\title{
Presynapses in Kenyon Cell Dendrites in the Mushroom Body Calyx of Drosophila
}

\author{
Frauke Christiansen, ${ }^{1,4 \star}$ Christina Zube, ${ }^{1 \star}$ Till F. M. Andlauer, ${ }^{1,4,5,6}$ Carolin Wichmann, ${ }^{1,4}$ Wernher Fouquet, ${ }^{1,4}$ \\ David Owald, ${ }^{1,4}$ Sara Mertel, ${ }^{1}$ Florian Leiss, ${ }^{2}$ Gaia Tavosanis, ${ }^{2}$ Abud J. Farca Luna, ${ }^{3}$ Andre Fiala, ${ }^{3}$ and Stephan J. Sigrist ${ }^{1,4}$ \\ ${ }^{1}$ Department of Genetics, Institute for Biology, Freie Universität Berlin, 14195 Berlin, Germany, ${ }^{2}$ Department of Molecular Neurobiology, Dendrite \\ Differentiation Group, Max Planck Institute of Neurobiology, 82152 München-Martinsried, Germany, ${ }^{3}$ Department of Molecular Neurobiology of Behavior, \\ Georg-August-Universität Göttingen, 37077 Göttingen, Germany, ${ }^{4}$ NeuroCure Cluster of Excellence, Charité Berlin, 10117 Berlin, Germany, ${ }^{5}$ Rudolf \\ Virchow Center/Deutsche Forschungsgemeinschaft Research Center for Experimental Biomedicine, Universität Würzburg, 97080 Würzburg, Germany, and \\ ${ }^{6}$ Max Planck Institute for Colloids and Interfaces, Science Park Golm, 14424 Potsdam, Germany
}

Plastic changes at the presynaptic sites of the mushroom body (MB) principal neurons called Kenyon cells (KCs) are considered to represent a neuronal substrate underlying olfactory learning and memory. It is generally believed that presynaptic and postsynaptic sites of KCs are spatially segregated. In the MB calyx, KCs receive olfactory input from projection neurons (PNs) on their dendrites. Their presynaptic sites, however, are thought to be restricted to the axonal projections within the MB lobes. Here, we show that KCs also form presynapses along their calycal dendrites, by using novel transgenic tools for visualizing presynaptic active zones and postsynaptic densities. At these presynapses, vesicle release following stimulation could be observed. They reside at a distance from the PN input into the $\mathrm{KC}$ dendrites, suggesting that regions of presynaptic and postsynaptic differentiation are segregated along individual $\mathrm{KC}$ dendrites. KC presynapses are present in $\gamma$-type KCs that support short- and long-term memory in adult flies and larvae. They can also be observed in $\alpha / \beta$-type KCs, which are involved in memory retrieval, but not in $\alpha^{\prime} / \beta^{\prime}$-type KCs, which are implicated in memory acquisition and consolidation. We hypothesize that, as in mammals, recurrent activity loops might operate for memory retrieval in the fly olfactory system. The newly identified KC-derived presynapses in the calyx are, inter alia, candidate sites for the formation of memory traces during olfactory learning.

\section{Introduction}

Animals detect odors through olfactory sensory neurons. The odor information is processed by a primary olfactory center, constituted by the antennal lobe in insects (Stocker et al., 1983) or the olfactory bulb in vertebrates (Mombaerts, 2001). Subsequently, olfactory information is conveyed to secondary centers via olfactory projection neurons (PNs) in insects or mitral/tufted cells in vertebrates. The olfactory nervous systems of insects and mammals are comparable in their anatomical organization, suggesting that fundamentals of olfaction and olfactory learning might be similar (Davis, 2004). In the relatively simple Drosophila brain, PNs target two separate neuropils, the lateral horn and the mushroom body (MB) (Strausfeld, 1976). In the MB calyx, PNs con-

\footnotetext{
Received Dec. 15, 2010; revised April 14, 2011; accepted April 28, 2011

Author contributions:F.C. and S.J.S. designed research; F.C., C.Z., C.W., W.F., D.O., S.M., F.L., G.T., A.J.F.L., and A.F. performed research; A.F. and S.J.S. contributed unpublished reagents/analytic tools; F.C., C.Z., T.F.M.A., A.J.F.L., and A.F. analyzed data; F.C., C.Z., and S.J.S. wrote the paper.

This work was supported by Deutsche Forschungsgemeinschaft Grants SFB 554 and EXC 257. We thankE. Buchner for the Nc82 antibody, A. Thum for pCaSpeR::mb247, G. Miesenböck for UAS-synapto-pHluorin, M. Heisenberg for h24-GAL4, B. Mentzel and T. Raabe for the MARCM lines, and F. Zehe and C. Quentin for technical assistance. We are grateful to M. Heisenberg, R. Kittel, A. Thum, E. Buchner, H. Tanimoto, and 0. Khorramshahi for fruitful discussions and valuable suggestions.

*F.C. and C.Z. contributed equally to this work.

Correspondence should be addressed to Stephan J. Sigrist, Department of Genetics, Institute for Biology, Freie Universität Berlin, 14195 Berlin, Germany. E-mail: stephan.sigrist@fu-berlin.de.

DOI:10.1523/JNEUROSCI.6542-10.2011

Copyright $\odot 2011$ the authors $\quad 0270-6474 / 11 / 319696-12 \$ 15.00 / 0$
}

nect to Kenyon cells (KCs) via large cholinergic boutons that contact claw-like endings of the KC dendrites (Schürmann, 1974; Yasuyama et al., 2002; Ramaekers et al., 2005). Several behavioral and genetic studies in different insect species have demonstrated that the MBs play an essential role in olfactory associative learning (for review, see Heisenberg, 1998, 2003; Roman and Davis, 2001; Waddell and Quinn, 2001a; Dubnau et al., 2003; Gerber et al., 2004; Davis, 2005; Keene and Waddell, 2007). Moreover, they are involved in other complex behavioral functions such as sleep/ wake behavior, visual learning, courtship conditioning, and decision making (Liu et al., 1999; McBride et al., 1999; Joiner et al., 2006; Pitman et al., 2006; Yang et al., 2008; Miller et al., 2011). Not only KCs are involved in olfactory learning and memory, but also a number of MB-extrinsic neurons have been shown to play important roles (Hammer and Menzel, 1995, 1998; Waddell et al., 2000; Yu et al., 2004; Thum et al., 2007; Claridge-Chang et al., 2009; Krashes et al., 2009; Liu and Davis, 2009; Aso et al., 2010). The anatomical organization of the $\mathrm{MB}$ as well as its connectivity to input and output neurons are not completely understood. However, a precise description of the connectivity of the $\mathrm{MB}$ is a prerequisite for understanding the neuronal basis of MB-derived behaviors.

In this study, we investigated the organization of $\mathrm{KC}$ and $\mathrm{PN}$ synapses within the MB calyx. In contrast to previous assumptions, dendrites of certain subpopulations of KCs $(\gamma$ and $\alpha / \beta$ cells) formed presynaptic active zones (AZs). Presynaptic AZs are 
the regions of synaptic vesicle (SV) fusion. The distribution of these KC presynaptic sites in the calyx followed a defined pattern; they were spatially separated from the sites of cholinergic input onto KCs, provided by PNs. These KC-specific presynaptic sites could be stimulated to release SVs. Our study adds another degree of complexity to the olfactory circuit of Drosophila.

\section{Materials and Methods}

Animals. The following fly stocks were used: c305a-GAL4 (Krashes et al., 2007), c739-GAL4 (O’Dell et al., 1995; Yang et al., 1995), gh146-GAL4 (Stocker et al., 1997), h24-GAL4 (Zars et al., 2000), ok107-GAL4 (Connolly et al., 1996), UAS-brp-RNAi (Wagh et al., 2006, recombination of B3 and C8), UAS-synapto-pHluorin (Ng et al., 2002), UAS-Da $7^{G F P}$ (Leiss et al., 2009), UAS-brp-short ${ }^{m \text { Cherry }}$ (Schmid et al., 2008), UAS-syt ${ }^{\text {GFP }}$ (Zhang et al., 2002), mb247::brp-short ${ }^{G F P}$, w, hsFlp,UAS-mCD8-GFP;FRT82B,tubGAL80/ TM3,Sb;ok107-GAL4 (L. Luo, Howard Hughes Medical Institute, Stanford University, Palo Alto, CA), $w^{-}$;FRT82B (Lee and Luo, 2001). All fly strains were reared under standard laboratory conditions (Sigrist et al., 2003) at $25^{\circ} \mathrm{C}$.

Molecular cloning. We generated transgenic flies carrying the eGFPtagged brp-short construct under direct control of the $\mathrm{MB}$ enhancer mb247 (Schulz et al., 1996). brp-short corresponds to amino acids 4731226 of the 1740 aa Bruchpilot (BRP) protein (Schmid et al., 2008). brp-short ${ }^{G F P}$ was inserted in the pCaSpeR vector carrying the $m b 247$ enhancer in front of a minimal promoter from a heat shock gene (Schulz et al., 1996), using the StuI and the XbaI restriction sites of the vector and the SpeI and HpaI sites of the insert.

Dissection and immunohistochemistry. Adult and larval brains were dissected in ice-cold hemolymph-like saline (HL3) solution, fixed for 20 min in $4 \%$ paraformaldehyde in PBS, $\mathrm{pH} 7.2$, and then blocked in $10 \%$ normal goat serum (NGS) in PBS with $0.3 \%$ Triton X-100 (PBT) for 20 min. Adult and larval brains were incubated with primary antibodies together with $5 \%$ NGS overnight at room temperature and then washed in PBT for $3 \mathrm{~h}$, followed by overnight incubation with secondary antibodies at $4^{\circ} \mathrm{C}$. The brains were then washed for $3 \mathrm{~h}$ with $0.3 \mathrm{PBT}$ and mounted in VectaShield (Vector Laboratories) on slides. Adult brains stained with $\mathrm{Nc} 82$ (BRP ${ }^{\mathrm{Nc} 82}$ ) (Wagh et al., 2006) were incubated with $1 \%$ PBT for 30 min after fixation and were then incubated with the primary antibodies together with 5\% NGS for $48 \mathrm{~h}$ at room temperature. Afterward, the brains were washed for $5 \mathrm{~h}$ with PBT.

Antibody concentrations. Antibody concentrations were as follows: mouse $\mathrm{BRP}^{\mathrm{Nc82}}$, 1:100; rabbit anti-DSyd-1, 1:500 (Owald et al., 2010); rabbit anti-DsRed (BD Biosciences), 1:500; rabbit anti-GFP (Invitrogen), 1:500; goat anti-mouse Alexa 488 (Invitrogen; A11001), 1:500; goat anti-rabbit Cy3 (Dianova; 111-167-003), 1:500; goat anti-mouse Cy5 (Dianova; 111-177-003), 1:500.

Image acquisition. Conventional confocal images were acquired with a Leica TCS SP5 confocal microscope (Leica Microsystems) using a $63 \times$, 1.4 NA oil objective for calyx scans and a $20 \times, 0.7 \mathrm{NA}$ oil objective for whole-brain overview scans. Voxel size was $71 \times 71 \times 200 \mathrm{~nm}$ for calyx imaging and $120 \times 120 \times 500 \mathrm{~nm}$ for whole-brain imaging. Confocal stacks were processed using ImageJ software (http://rsbweb.nih.gov/ij/).

BRP-short can form agglomerations within the somata of the cells it is expressed in. As the KC somata are located very close to the calyx neuropil, we manually removed them in maximum-intensity projections (see Fig. $7 M-O)$ for clarity.

Analysis of the RNAi experiment. Flies expressing UAS-brp-RNAi under the control of ok107-GAL4 as well as $w 1118$ controls were raised in $68 \mathrm{ml}$ standard vials with a constant $12 \mathrm{~h}$ light/dark regimen. Adult female flies (2-5 $\mathrm{d}$ after eclosion) as well as female third-instar larvae were dissected.

$\mathrm{BRP}^{\mathrm{Nc} 82}$ and DSyd-1 signal intensities were quantified similarly as described by Kremer et al. (2010). Calyces were segmented using the Fiji (ImageJA, version 1.44) plug-in Segmentation Editor (http://pacific.mpicbg.de/). The images were then analyzed in Bitplane Imaris $64 \times$, version 6.23. The masks created in Fiji were used as a template for isolating the calyces in Imaris. Subsequently, the overall surface of the BRP ${ }^{\mathrm{Nc} 82}$ and DSyd-1 channels within the calyx was determined. In addition, number and surface areas of the individual BRP ${ }^{\mathrm{Nc} 82}$ and DSyd-1 spots were identified, as well as the respective intensities within each spot. For this, the
Imaris surface tool was used, using seed detection and region growing algorithms. The data were further analyzed using Microsoft Office 2008 and StatSoft Statistica 9.1.

Mosaic analysis with a repressible cell marker. Genotype of flies with mCD8-GFP and D $\alpha 7^{\text {mCherry }}$ coexpressing clones was as follows: $w$, hsFlp $/ w^{-}$; UAS-mcd8-GFP/UAS-D $\alpha 7^{\text {mCherry }}$ FRT82b, tubGAL80/ neoFRT82b; ok107-GAL4/+. Genotype of flies with mCD8-GFP and $B R P$-short ${ }^{m \text { Cherry }}$ coexpressing clones was as follows: $w, h s F l p / w^{-}$; UAS-mcd8GFP/UAS-brp-short ${ }^{\text {mCherry }}$; FRT82b, tubGAL80/neoFRT82b; ok107-Gal4/+.

Vials containing female and male eggs, first-, second-, or third-instar larvae or pupae were transferred into a $37^{\circ} \mathrm{C}$ water bath for $30 \mathrm{~min}$. Together, we scanned 89 calyces. The clones depicted belong to the $\alpha / \beta$ $\mathrm{KC}$ subpopulation. For the clone expressing $\mathrm{D} \alpha 7$ shown in Figure 6, $I$ and $J$, the heat shock was applied $5 \mathrm{~d}$ before pupal eclosion. For the clone expressing BRP-short shown in Figure 6, $K$ and $L$, the heat shock was applied $3 \mathrm{~d}$ before pupal eclosion.

Functional imaging. Flies were cooled on ice for several minutes and immobilized in a truncated $1 \mathrm{ml}$ pipette tip. This preparation was glued to a plastic coverslip (Plano) with dental glue (Protemp II; 3M ESPE). Subsequently, a window was cut into the head of the fly using a splint of a razor blade and a blade holder. Trachea and fat bodies were carefully removed to expose the brain. The brain was covered with Ringer's solution (5 mм HEPES, $130 \mathrm{~mm} \mathrm{NaCl}, 5 \mathrm{~mm} \mathrm{KCl}, 2 \mathrm{~mm} \mathrm{MgCl}_{2}, 2 \mathrm{~mm} \mathrm{CaCl}_{2}$ ) as a physiological medium. For eliciting neuronal depolarization, $\mathrm{KCl}$ (final concentration of $100 \mathrm{~mm}$ ) was injected into the drop of Ringer's solution covering the brain. The imaging setup consisted of a fluorescence microscope (Axio Examiner D1; Zeiss), equipped with a xenon lamp (Lambda DG-4; Sutter Instrument), a 14 bit camera (Coolsnap HQ2; Photometrics), and a GFP filter set. Data acquisition was controlled by the software Metafluor (Visitron Systems). Images were acquired using a $40 \times$ water-immersion objective at a frame rate of $5 \mathrm{~Hz}$ and an excitation wavelength of $488 \mathrm{~nm}$. Image sequences were analyzed by choosing a region of interest (ROI) covering the calycal region. Average emission intensities from Synapto-pHluorin were quantified for each image. Average intensities of a ROI outside the labeled structure was subtracted as background. The emission intensity $(F)$ at the frame immediately before $\mathrm{KCl}$ application was determined as $F_{0}$, and $\Delta F / F_{0}$ was calculated for each image. The software Origin, version 8.1 (OriginLab), was used for data evaluation. For illustrating the entire MB (see Fig. $5 B$ ), images were acquired at $1 \mu \mathrm{m}$ steps in the $Z$ direction. A maximumintensity $Z$ projection was generated from the entire stack.

\section{Results}

\section{KC-derived presynapses within the MB calyx of Drosophila adults and larvae}

We aimed at characterizing $\mathrm{KC}$ presynapses in the $\mathrm{MB}$ using a detailed molecular-anatomical approach. Presynapses are characterized by AZs containing discrete electron-dense specializations of roughly uniform size, called T-bars in Drosophila (Prokop and Meinertzhagen, 2006; Wichmann and Sigrist, 2010). The large scaffolding protein BRP is an essential component of T-bars (Kittel et al., 2006; Wagh et al., 2006). To label AZs of KCs, we expressed a fragment of BRP (BRP-short) fused to GFP (BRPshort $^{\mathrm{GFP}}$ ) specifically in KCs. BRP-short ${ }^{\mathrm{GFP}}$ colocalizes clearly with endogenous BRP (Schmid et al., 2008) but is not detected by the monoclonal $\alpha \mathrm{BRP}$ antibody $\mathrm{Nc} 82\left(\mathrm{BRP}^{\mathrm{Nc} 82}\right)$, which binds to an epitope close to the BRP C terminus (Wagh et al., 2006). Notably, BRP-short ${ }^{\text {GFP }}$ depends on endogenous BRP for AZ localization (S. J. Sigrist, unpublished observations). Therefore, $\mathrm{BRP}$-short labels only preexisting AZs, characterized by the presence of endogenous full-length BRP, which is recognized by $\mathrm{BRP}^{\mathrm{Nc} 82}$. In addition, a previous study showed that the number of $\mathrm{BRP}^{\mathrm{Nc} 82}$-positive puncta is not modified upon expression of BRP-short (Kremer et al., 2010).

To specifically label KC-derived AZs, the KC-specific enhancer mb247 (Schulz et al., 1996) was fused to the BRP-short GFP open reading frame. As expected, MB lobes of both larvae (Fig. 

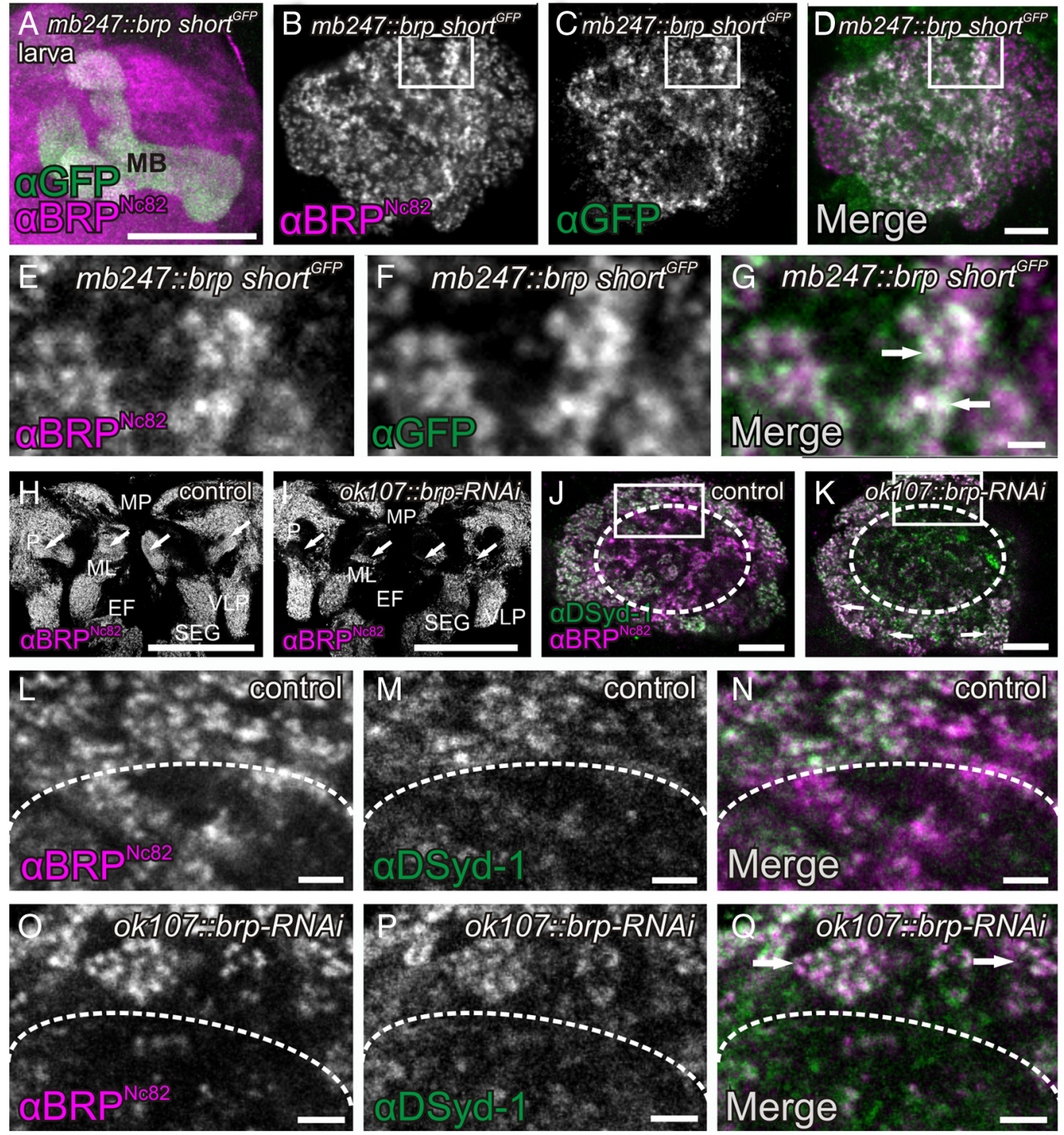

Figure 1. Evidence for KC-derived presynapses within the MB calyx of Drosophila larvae. $A-G$, Expression of the BRP fragment BRP-short ${ }^{\text {GFP }}$ under control of the $K C$ enhancer $m b 247$ reveals a strong signal in the MB lobes (A, maximum-intensity projection) and the calyx of the larva $(\boldsymbol{C}, \boldsymbol{F})$. Costaining with BRP ${ }^{\mathrm{N} 882}$, an antibody against the presynaptic AZ protein BRP (A, mushroom body; $\boldsymbol{B}, \boldsymbol{E}$, calyx), showed a clear overlap with the KC-derived BRP-short ${ }^{\text {GFP }}$ signal in the calyx ( $\boldsymbol{D}, \boldsymbol{G}$, arrows), suggesting the existence of a KC-derived population of AZs. $\boldsymbol{E}-\boldsymbol{G}$, Cutout of the calyx shown in $\boldsymbol{B}-\boldsymbol{D}$ in a higher magnification. $\boldsymbol{H}-\mathbf{Q}$, UAS-brp-RNAi expressed in KCS (ok107-GAL4) provoked a strong reduction of the BRP ${ }^{\text {NC82 }}$ label in both MB lobes and calyx. $\boldsymbol{H}, \boldsymbol{I}$, Larval brain overview. In the control brain stained with $\alpha \mathrm{BRP}^{\mathrm{Nc} 82}$, major neuropils are clearly visible, including the mushroom body lobes (H, arrows). UAS-brp-RNAi expressed in KCs resulted in a strong reduction of the MB lobe label $\left(\boldsymbol{I}\right.$, arrows). $\boldsymbol{J}, \boldsymbol{K}$, Colabeling of $\alpha$ BRP ${ }^{\mathrm{N} C 82}$ together with an antibody against the AZ protein DSyd1 showed a specific effect of the expression of UAS-brp-RNAi in KCs on the BRP label in the calyx. Reduction of BRP was confined to the regions in the center of the calyx ( $\boldsymbol{K}$, dotted circle) but did not occur in areas where KCs are postsynaptic to PN presynaptic terminals, the macroglomeruli $(\boldsymbol{K}$, arrows). $\boldsymbol{L}-\mathbf{Q}$, Higher magnification views of calycal regions shown in $\boldsymbol{J}$ and $\boldsymbol{K}$, marked in $\boldsymbol{J}$ and $\boldsymbol{K}$ by white rectangles. While the BRP label is clearly reduced $(\boldsymbol{O}, \boldsymbol{Q}$, below the dotted line) compared with the control $(\boldsymbol{L}, \boldsymbol{N})$, the DSyd1 label remains unaffected $(\boldsymbol{M}, \boldsymbol{P})$. The macroglomeruli also show no reduction in BRP label $(\boldsymbol{Q}$, arrows). ML, Medial lobe; $\boldsymbol{P}$, peduncle; MP, medial protocerebrum; EF, esophageal foramen; VLP, ventrolateral protocerebrum; $\mathrm{SEG}$, subesophageal ganglion; $M B$, mushroom body. All images show single optical slices, except when stated differently. Slice thickness, $200 \mathrm{~nm}$. Scale bars: $\boldsymbol{A}, \boldsymbol{H}, \boldsymbol{I}, 50 \mu \mathrm{m} ; \boldsymbol{B}-\boldsymbol{D}, \boldsymbol{J}, \boldsymbol{K}, 10 \mu \mathrm{m} ; \boldsymbol{E}-\mathbf{G}, 5 \mu \mathrm{m} ; \boldsymbol{L}-\mathbf{Q}, 2 \mu \mathrm{m}$.

$1 A$ ) and flies (Fig. 2A) were clearly labeled. Consistent with the expression previously reported for the mb247 enhancer (Riemensperger et al., 2005; Krashes et al., 2007), $\gamma, \alpha / \beta$, and $\alpha^{\prime} / \beta^{\prime}$ neurons were labeled by this construct (data not shown). In $m b 247:: b r p$-short ${ }^{G F P}$ animals, BRP-short ${ }^{\mathrm{GFP}}$ was arranged in distinct puncta, which were also labeled by BRP ${ }^{\mathrm{Nc} 82}$ (Figs. 1A, 2A). This indicates that endogenous BRP is present at these AZs. In our study, we used mb247::brp-short ${ }^{G F P}$ for the identification of KC-derived AZs.

To a minor degree, $m b 247:: b r p$-short ${ }^{G F P}$ drives expression in MB-extrinsic neurons. It is therefore possible that the synapses we are looking at belong to MB-extrinsic neurons. To exclude this possibility, we confirmed our results by checking UAS-brpshort ${ }^{m \text { Cherry }}$ expression in several different MB GAL4-lines [ok107-GAL4, h24-GAL4, c739-GAL4, c305a-GAL4 (see Fig. $7 A-O)$ and $m b 247-G A L 4,201 y$-GAL4, $d 52 h$-GAL4, $1471 y$-GAL4 (data not shown)].

Surprisingly, a clear BRP-short ${ }^{\text {GFP }}$ label was also present in the calyces of both larvae (Fig. 2C,D, F, G) and adult flies (Fig. $1 C, D, F, G)$. This label consisted of discrete puncta, typical for individual AZs, which were also labeled by $\mathrm{BRP}^{\mathrm{Nc} 82}$ (Figs. $1 G$, arrows; $2 D, G$, arrows). We observed these puncta within calyces of both female and male animals, in second- and third-instar larvae, and throughout the life span from newly eclosed to 14-d- 

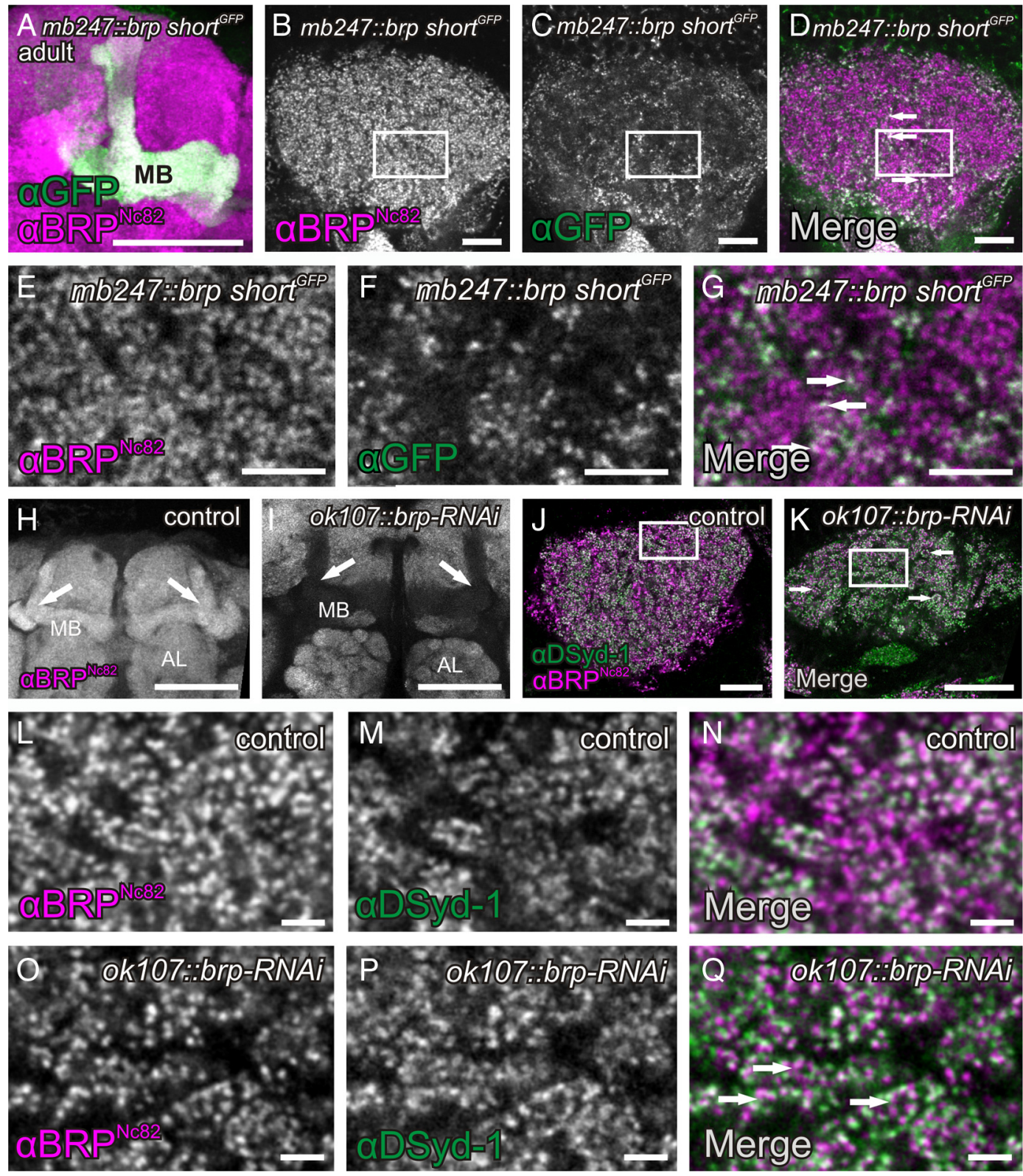

Figure 2. Evidence for KC-derived presynapses within the MB calyx of Drosophila adults. $A-G$, Expression of the BRP fragment BRP-short ${ }^{\text {GFP }}$ under control of the KC enhancer $m b 247$ reveals a strong signal in MB lobes ( $\boldsymbol{A}$, maximum-intensity projection) and calyces of adult flies ( $\boldsymbol{C}$; higher magnification in $\boldsymbol{F}$ ). The costaining of the presynaptic AZ protein BRP ( $\boldsymbol{B}$; high magnification in $\boldsymbol{E}$ ) showed a clear overlap with the KC-derived BRP-short ${ }^{G F P}$ signal ( $\boldsymbol{D}$; higher magnification in $\mathbf{G}$; arrows), suggesting the existence of a KC-derived population of AZs. $\boldsymbol{H}$-Q $\mathbf{Q}$, UAS-brp-RNAi expressed in KCs (ok107-GAL4) provoked a strong reduction of the BRP ${ }^{N(82}$ label in both MB lobes $(\boldsymbol{H}, \boldsymbol{I}$, arrows) and calyces $(\boldsymbol{J}, \boldsymbol{K}$, arrows; higher magnification in $\boldsymbol{L}$ and $\boldsymbol{O})$. In the calyx, the reduction of BRP was confined to the regions outside of microglomeruli ( $\boldsymbol{K}$, arrows). For a higher magnification, compare $\boldsymbol{L}-\boldsymbol{Q}$. Since $\alpha$ DSyd-1 localization at AZs was shown to be independent of BRP (0wald et al., 2010), we used this marker as an independent reference. DSyd-1 labeling remained unaffected from the brp-RNAi expression in KCs $(M, P)$. MB, Mushroom body; AL, antennal lobe. All images show single optical slices, except when stated differently. Slice thickness, $200 \mathrm{~nm}$. Scale bars: $\boldsymbol{A}, \boldsymbol{H}, \boldsymbol{I}, 50 \mu \mathrm{m} ; \boldsymbol{B}-\boldsymbol{D}, \boldsymbol{K}, 10 \mu \mathrm{m} ; \boldsymbol{E}-\mathbf{G}, 5 \mu \mathrm{m} ; \boldsymbol{L}-\mathbf{Q}, 2 \mu \mathrm{m}$.

old adult flies (data not shown). Furthermore, we confirmed these observations by expressing BRP-short ${ }^{\mathrm{GFP}}$ and BRPshort ${ }^{\text {mCherry }}$ with several independent GAL4-KC drivers (see Fig. $7 A-O)$ (data not shown). This result is surprising as $\mathrm{KC}$ dendrites were so far considered to be exclusively postsynaptic (Yasuyama et al., 2002).

It might be argued that the ectopic expression of BRPshort ${ }^{\mathrm{GPP}}$ could artificially cluster endogenous BRP and thereby mimic natural presynaptic AZs. To add further proof to the hypothesis that KCs express presynapses in the calyx and that BRPshort ${ }^{\text {GFP }}$ highlights only already existing AZs, we attempted to reduce BRP levels by expressing brp-directed RNA interference constructs (UAS-brp-RNAi) specifically within KCs. We have demonstrated that this construct could be used to strongly reduce BRP levels in the eyes of adult flies as well as in larval motoneurons in a previous experiment (Wagh et al., 2006). For this RNAi experiment, we used an antibody against the presynaptic AZ protein DSyd-1 as an AZ marker independent of BRP. We characterized DSyd-1 in a previous study (Owald et al., 2010). DSyd-1 incorporation precedes $\mathrm{BRP}$ incorporation during $\mathrm{AZ}$ formation and also persists in $b r p$ mutants. UAS-brp-RNAi was expressed under control of the broad KC driver line ok107-GAL4. Expression of the RNAi clearly reduced $\mathrm{BRP}^{\mathrm{Nc} 82}$ label in MB lobes and calyces of both adults (Fig. $1 H-Q$ ) and larvae (Fig. $2 H-Q$ ). We confirmed this observation by quantification (Fig. 3, Table 1). For this quantification, we used the 3D software Imaris to calcu- 

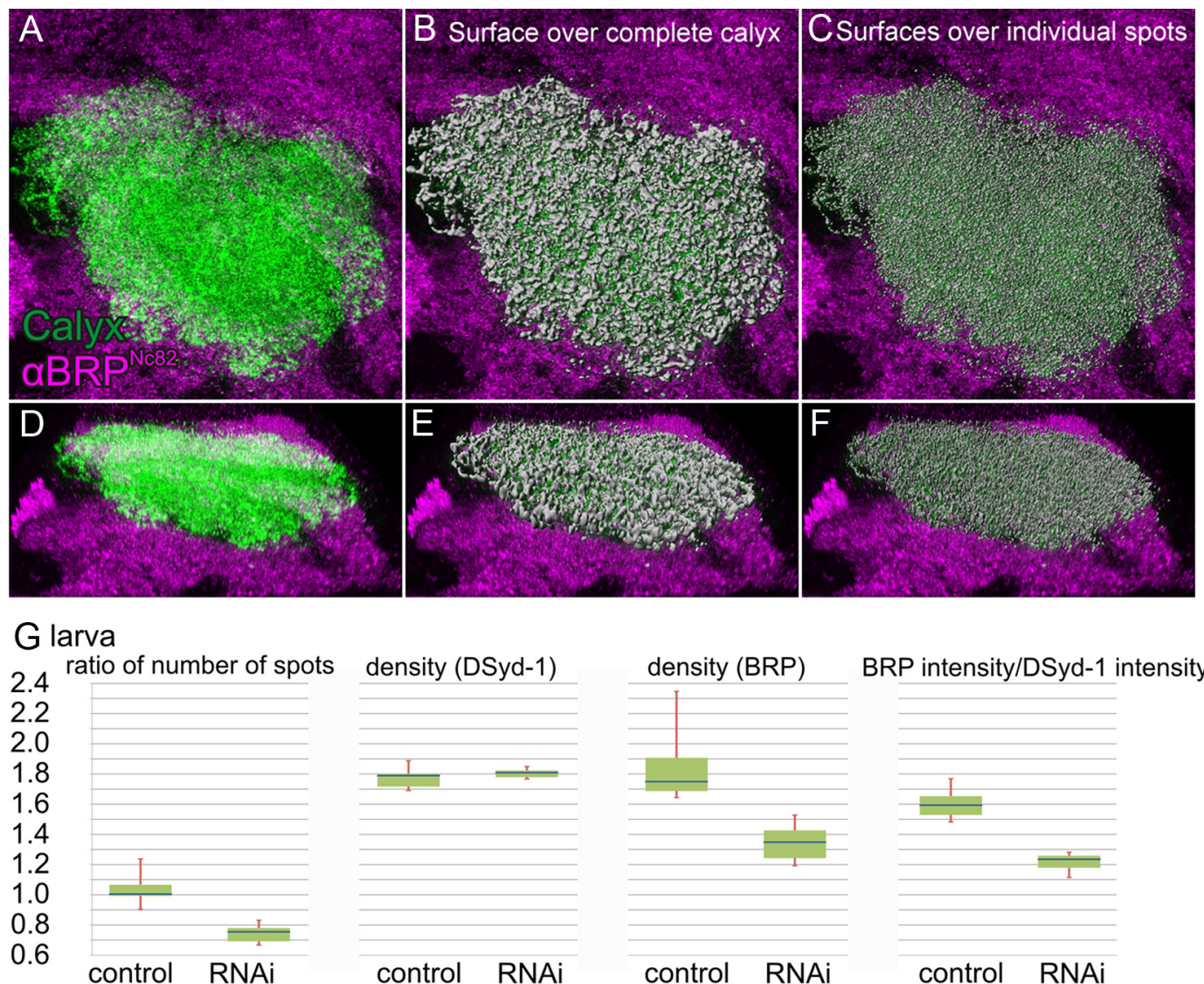

BRP intensity/DSyd-1 intensity

$\mathrm{H}$ adult
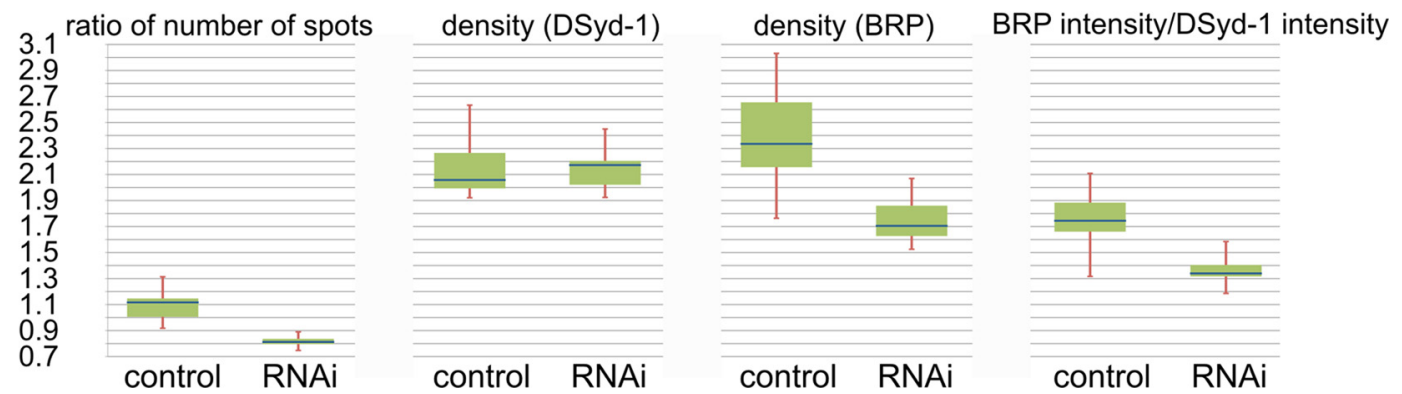

Figure 3. Segmentation and quantification of BRP ${ }^{\mathrm{N} 882}$ signal within the calyx, with and without expression of UAS-brp-RNAiin $K C S$. $A-F$, Visualization of the segmentation and $3 D$ reconstruction of the calyx used for quantification. $\boldsymbol{A}-\boldsymbol{C}$, Top view. $\boldsymbol{D}-\boldsymbol{F}$, Side view. $\boldsymbol{A}, \boldsymbol{D}$, Segmented calyx (green) within the BRP ${ }^{\mathrm{N} 882}$ staining labeling all neuropils (magenta). $\boldsymbol{B}, \boldsymbol{E}$, Computed 3D surface covering the complete $B R P^{N(82}$-positive signal in the calyx (gray), superimposed on the staining shown in $\boldsymbol{A}$ and $\boldsymbol{D}$. $\boldsymbol{C}, \boldsymbol{F}$, Three-dimensional surfaces of individual BRP ${ }^{\mathrm{N}(82}$-positive spots (gray), superimposed on the staining shown in $A$ and $\boldsymbol{D}$. The surfaces used for the detection of the number of AZs. For additional detailed images of such a 3 D reconstruction of the BRP ${ }^{\mathrm{N} 882}$ signal, also see Kremer et al. (2010). $\mathbf{G}, \boldsymbol{H}$, Results of the quantification. Blue bar, Median; green box, interquartile range; red whiskers, $\min /$ max values. The Mann-Whitney $U$ test was used to determine two-sided exact $p$ values. $G$, Larval calyces, $n=6$ animals for both genotypes. Ratio of number of spots is the ratio of BRP ${ }^{N(82}$-positive spots versus DSyd-1-positive spots, $p=0.002$. Density is the number of spots per calyx area. DSyd- 1 density, $p=0.485 ; B R P^{N<82}$ density, $p=0.002$. BRP intensity/DSyd-1 intensity is the ratio of the BRP ${ }^{N}$ (82 signal intensity versus the DSyd-1 signal intensity within a surface covering the complete calyx, $p=0.002$. $\boldsymbol{H}$, Adult calyces, $n=10$ animals for brp-RNAiand $n=9$ for controls. Ratio of number of spots, $p<0.001$. DSyd-1 density, $p=0.968 ;$ BRP $^{\mathrm{N} 82}$ density, $p=0.001$. Ratio of signal intensity, $p=0.002$.

Table 1. Change in percentage of the indicated parameters between brp-RNAi and control animals

\begin{tabular}{lllll}
\hline & $\begin{array}{l}\text { Ratio of number of spots } \\
\left(\text { BRP }^{\text {Nc82 } / D S y d-1) ~}\right.\end{array}$ & $\begin{array}{l}\text { Spot density } \\
\left(\text { BRP N }^{\text {N82 })}\right.\end{array}$ & $\begin{array}{l}\text { Spot density } \\
\text { (DSyd-1) }\end{array}$ & $\begin{array}{l}\text { Ratio of intensity } \\
\left(\text { BRP }^{\text {Nc82 } / D S y d-1) ~}\right.\end{array}$ \\
\hline Adults & -27.2 & -26.9 & +5.6 & -23.1 \\
Larvae & -24.8 & -22.9 & +1.1 & -22.4 \\
\hline
\end{tabular}

For additional details, see Figure $3, G$ and $H$, and Results.

late $3 \mathrm{D}$ surfaces of calyces, based on $\mathrm{BRP}^{\mathrm{Nc} 82}$ and DSyd-1 signals (Fig. $3 A-F$ ). In this manner, we determined the number of spots positive for $\mathrm{BRP}^{\mathrm{Nc} 82}$ as well as for the independent marker DSyd-1. Here, individual spots correspond to individual presynaptic AZs
(Kremer et al., 2010). In addition, we calculated the average intensities of the staining of the two markers within the calyx as well as the overall surface areas of $\mathrm{BRP}^{\mathrm{Nc82}}$ - and DSyd-1-positive signals in the calyx. From these data, we determined four parameters, summarized in Table 1 and Figure 3, $G$ and $H$. The results show that the DSyd-1 signal remained unaffected upon expression of the RNAi, while BRP levels were reduced, in the number of spots as well as in the staining intensity. The finding that the DSyd-1 signal was not reduced is consistent with the observation that the localization of DSyd-1 at AZs is independent of BRP. In view of these data, we conclude that KCs form presynaptic AZs within the calyx. In the following, we name these KC-derived AZs in the calyx, short KCACs. 

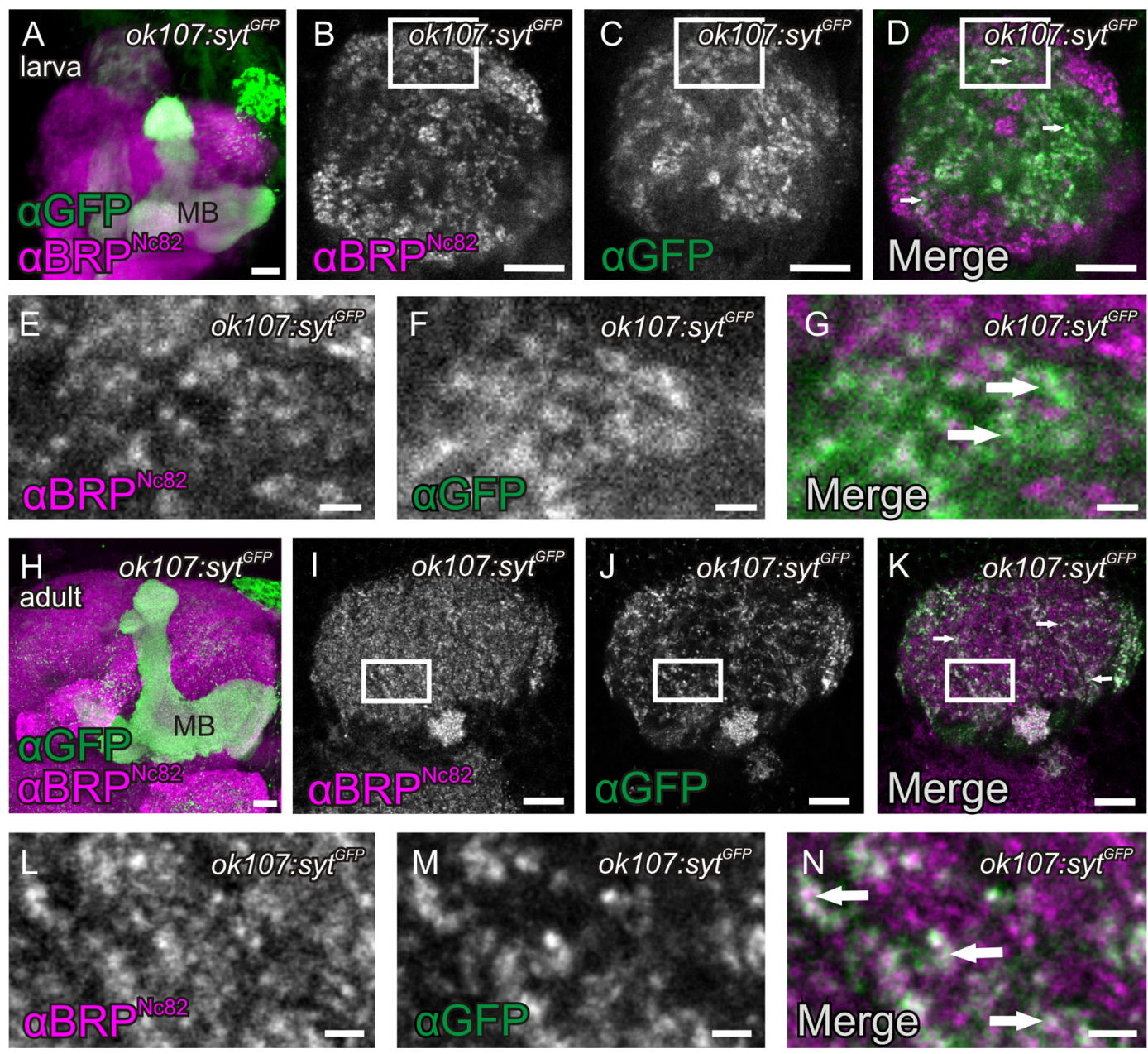

Figure 4. Presence of SVs in KCACS. $\boldsymbol{A}-\boldsymbol{H}$, Expression of a GFP-fusion of the SV marker Synaptotagmin (syt ${ }^{\text {GFP }}$ ) with the KC driver ok107-GAL4 in MB lobes and calyces of larvae $(\boldsymbol{A}$, MB lobes, maximum-intensity projection; $\boldsymbol{B}-\boldsymbol{G}$, calyx) and adult flies ( $\boldsymbol{H}$, MB lobes, maximum-intensity projection; $\boldsymbol{I}-\boldsymbol{N}$, calyx). Costaining with BRP ${ }^{\mathrm{Nc82}}$ revealed that syt ${ }^{\mathrm{GFP}}$ expressed in $K C \mathrm{~S}$ locates between microglomerular and macroglomerular complexes ( $\boldsymbol{B}-\boldsymbol{D}$, arrows; $\boldsymbol{I}-\boldsymbol{K}$, arrows). For a higher magnification in larvae, compare $\boldsymbol{E}-\boldsymbol{G}$ (arrows), and for adults, $\boldsymbol{L}-\boldsymbol{N}$ (arrows). All images show single optical slices, except when stated differently. Slice thickness, $200 \mathrm{~nm}$. MB, Mushroom body. Scale bars: $\boldsymbol{A}, \boldsymbol{B}-\boldsymbol{D}, \boldsymbol{H}-\boldsymbol{K}, 10 \mu \mathrm{m} ; \boldsymbol{E}-\mathbf{G}, \boldsymbol{L}-\boldsymbol{N}, 1 \mu \mathrm{m}$.

PNs contact KCs in structures called macroglomeruli (in larvae) (Ramaekers et al., 2005) or microglomeruli (in flies) (Schürmann, 1974; Yasuyama et al., 2002). Interestingly, only the $\mathrm{BRP}^{\mathrm{Nc82}}$ label outside microglomeruli or macroglomeruli was strongly reduced (Figs. $1 \mathrm{~K}$, dotted circle; $O, Q$, below dotted line; $2 K, O, Q)$. The $\mathrm{BRP}^{\mathrm{Nc} 82}$ immunoreactivity within microglomeruli or macroglomeruli, however, appeared unaffected (Figs. $1 K, O, Q$, arrows; $2 K, O, Q$, arrows). Notably, the quantification indicates a reduction of BRP levels in the calyx by $>20 \%$ in both adult flies and larvae (Table 1). Therefore, we estimate that KCderived AZs make up $>20 \%$ of all AZs within the calyx.

\section{Activity-mediated vesicle cycling at KC-derived AZs in}

the calyx

So far, we assayed proteins specific and indicative for the $\mathrm{AZ}$ cytomatrix (BRP, DSyd-1) to demonstrate the existence of KCderived presynaptic AZs. Next, we tested whether markers for SVs were associated with KCACs as well. To this end, we expressed a GFP-fusion to the presynaptic vesicle protein Synaptotagmin (UAS-syt $t^{G F P}$ ) (Zhang et al., 2002) under control of the KC driver ok107-GAL4. Strong signals in both MB lobes (Fig. $4 A, H$ ) and calyx (Fig. $4 C, D, F, G, J, K, M, N$ ) were observed. While the Synaptotagmin label was less spatially confined than the AZ cytomatrix label (BRP ${ }^{\mathrm{Nc82}}$ ) (Fig. $\left.4 B-G, I-N\right)$, syt ${ }^{\mathrm{GFP}}$ ex- pressed from KCs also clearly located in between macroglomeruli (data not shown) and microglomeruli (Fig. $4 K, N$, arrows).

To test whether the KCACs are capable of SV release, we made use of functional optical imaging of an in vivo preparation (Fig. $5 A$ ) to visualize Synapto-pHluorin ( $\mathrm{Ng}$ et al., 2002) expressed in KCs by ok107-GAL4 (Fig. 5B). Synapto-pHluorin consists of the $\mathrm{SV}$ protein Synaptobrevin, fused at its lumenal C terminus to ecliptic pHluorin, a pH-sensitive mutant of GFP, which is nonfluorescent at a $\mathrm{pH}<6$ upon excitation at $470 \mathrm{~nm}$. After vesicle fusion with the $\mathrm{AZ}$ membrane, pHluorin is exposed to the higher $\mathrm{pH}$ value of the extracellular space, which enhances the fluorescence emission and yields a visible signal (Miesenböck et al., 1998). The calyx is spatially separated from the MB lobes region; therefore, Synapto-pHluorin expression can be selectively monitored in this neuropil. Fluorescence of Synapto-pHluorin could be observed in the calyx, again indicating the presence of $\mathrm{KC}$ derived SVs within the calyx (Fig. 5B). Stimulation with $\mathrm{KCl}$ causes a general depolarization of the brain, which leads to SV release. While imaging this release, the measurable signal is superimposed on a general decrease of visible label due to photobleaching. Nonetheless, upon depolarization, a strong increase in fluorescence in the calyx was observed (Fig. $5 C, D$ ). Thus, the presynaptic AZs derived from KCs in the calyx can release SVs. 
KCACs cluster distant from

PN::KC synapses

Endogenously present $\mathrm{BRP}^{\mathrm{Nc} 82}$-positive spots in the MB lobes were, to a large extent, also targeted by BRP-short expressed in KCs. In the calyx, however, only a fraction of the $\mathrm{BRP}^{\mathrm{Nc} 82}$-positive spots could be labeled by $\mathrm{KC}$-expressed BRP-short (Figs. $1 B-D, 2 B-D)$. This is expected, since a dominant input to the calyx is delivered by PNs (Yasuyama et al., 2002), and according to our quantification, only $\sim 20 \%$ of the calycal AZs are expected to correspond to KC-derived AZs (KCACs) (Table 1, Fig. 3). KCACs located in between macroglomeruli and microglomeruli. This suggests that KCACs remain segregated from the cholinergic $\mathrm{PN}: \mathrm{KC}$ synapses. To explicitly examine this, we introduced additional molecular markers. First, we sought to label the postsynaptic densities (PSDs) of KCs at their cholinergic input synapses. To this end, we fused the KC-specific enhancer $m b 247$ to the gene encoding for the $\mathrm{D} \alpha 7$ acetylcholine (ACh) receptor subunit (Grauso et al., 2002; Fayyazuddin et al., 2006) $\left(m b 247:: d \alpha 7^{G F P}\right)$, flanked by the $g f p$ gene. We have already previously used $\mathrm{D} \alpha 7^{\mathrm{GFP}}$ to label PSDs of cholinergic synapses (Leiss et al., 2009; Raghu et al., 2009; Kremer et al., 2010). The GFP signal of $m b 247:: d \alpha 7^{G F P}$ was absent from the MB lobes of larvae and adults (data not shown), indicating that, as expected, ACh receptors of $\mathrm{KCs}$ are restricted to the calyx region. Within the MB calyx, mb247:: $d \alpha 7^{G F P}$ produced a strong staining in macroglomeruli (in larvae) (data not shown) and microglomeruli (in adults) (Fig. 6A). The GFP label was arranged in little patches corresponding to postsynaptic sites, which were juxtaposed to individual PN AZs (Kremer et al., 2010). To visualize PN-derived AZs, we expressed UAS-brp-short ${ }^{\text {mCherry }}$ under the control of a broad PN driver ( gh146-GAL4) (Fig. 6B,F) together with $m b 247:: d \alpha 7^{G F P}$ (Fig. 6A). As expected, we observed presynaptic AZs of PNs juxtaposed to the cholinergic PSDs of the KCs in the adult calyx (Fig. 6C,D, arrows). In contrast, when we expressed BRP-short in KCs (mb247:: brp-short ${ }^{\text {GFP }}$ ) (Fig. 6E) together with UAS-brp-short ${ }^{\text {mCherry }}$ under control of the PN driver ( gh146-GAL4) (Fig. 6F), the KC-derived $\mathrm{BRP}$-short signals grouped away from the PN AZ populations (Fig. $6 G, H$, arrows).

In summary, covisualization of KCACs together with presynaptic AZs of PNs in larvae (data not shown) and adult flies underlined that the PN-derived AZs are primarily restricted to the microglomerular domains, while the KCACs reside within the intermicroglomerular regions.

\section{Segregation of presynaptic and postsynaptic domains within KC dendrites}

KCACs appeared clearly segregated from the cholinergic $\mathrm{PN}:$ KC synapses. Our analysis already suggested that both presynaptic and postsynaptic elements are formed by $\mathrm{KC}$ dendrites but are spatially segregated. For an analysis on the level of single neurons, we produced single $\mathrm{KC}$ clones using mosaic analysis with a repressible cell marker (MARCM) (Lee et al., 1999; Luo,
2007). While expression of mCD8::GFP allowed for visualizing the cell morphology, coexpression of BRP-short ${ }^{\text {mCherry }}$ served as a label for presynapses and coexpression of $\mathrm{D} \alpha 7^{\mathrm{mCherry}}$ served as a label for postsynapses. As expected (Yasuyama et al., 2002), $\mathrm{D} \alpha 7^{\mathrm{mCherry}}$ signal mainly clustered at the terminal claw-like regions of $\mathrm{KC}$ dendrites (Fig. 6I, J, arrows). Also, BRP-short ${ }^{\text {mCherry }}$ signal was observed in $\mathrm{KC}$ dendrites. However, we rarely found BRP-short ${ }^{\text {mCherry }}$ clusters directly at claws but rather at more proximal parts of the dendrites (Fig. $6 K, L$ ).

It might be argued that axons running back from the lobe region into the calyx might be responsible for the $\mathrm{KC}$-derived $\mathrm{AZ}$ label observed within the calyx. Yet in our single-cell analysis such axons were never observed. Instead, the physical segregation of presynaptic and postsynaptic specializations along KC dendrites could be further corroborated when we coexpressed BRPshort ${ }^{\text {mCherry }}$ and D $\alpha 7^{\mathrm{GFP}}$ using the broad KC driver ok107-GAL4 (Fig. $7 A-C$ ). We found that the BRP-short ${ }^{\text {mCherry }}$ signal was restricted to areas between the microglomeruli, visualized by $\mathrm{D} \alpha 7^{\mathrm{GFP}}$ (Fig. 7C, arrows). Thus, markers for presynaptic and postsynaptic specializations arrange separately when coexpressed within KCs dendrites. It thus appears likely that KC dendrites are of mixed identity and form presynaptic and postsynaptic stretches in consecutive, but hardly overlapping segments. For a schematic interpretation of these results, see Figure $6 \mathrm{M}$.

\section{The KC subtypes $\boldsymbol{\gamma}$ and $\boldsymbol{\alpha} / \boldsymbol{\beta}$, but not $\boldsymbol{\alpha}^{\prime} / \boldsymbol{\beta}^{\prime}$, form KCACs}

Depending on their axonal elaboration within the MB lobes, KCs are subgrouped into several classes $\left(\alpha / \beta, \alpha^{\prime} / \beta^{\prime}, \gamma\right)$ (Crittenden et al., 1998). To examine the contribution of specific KC subtypes to the calycal microcircuitry, KC subtype-specific GAL4 lines for $\gamma$ neurons [h24-GAL4 (Zars et al., 2000; Akalal et al., 2006)], $\alpha / \beta$ 

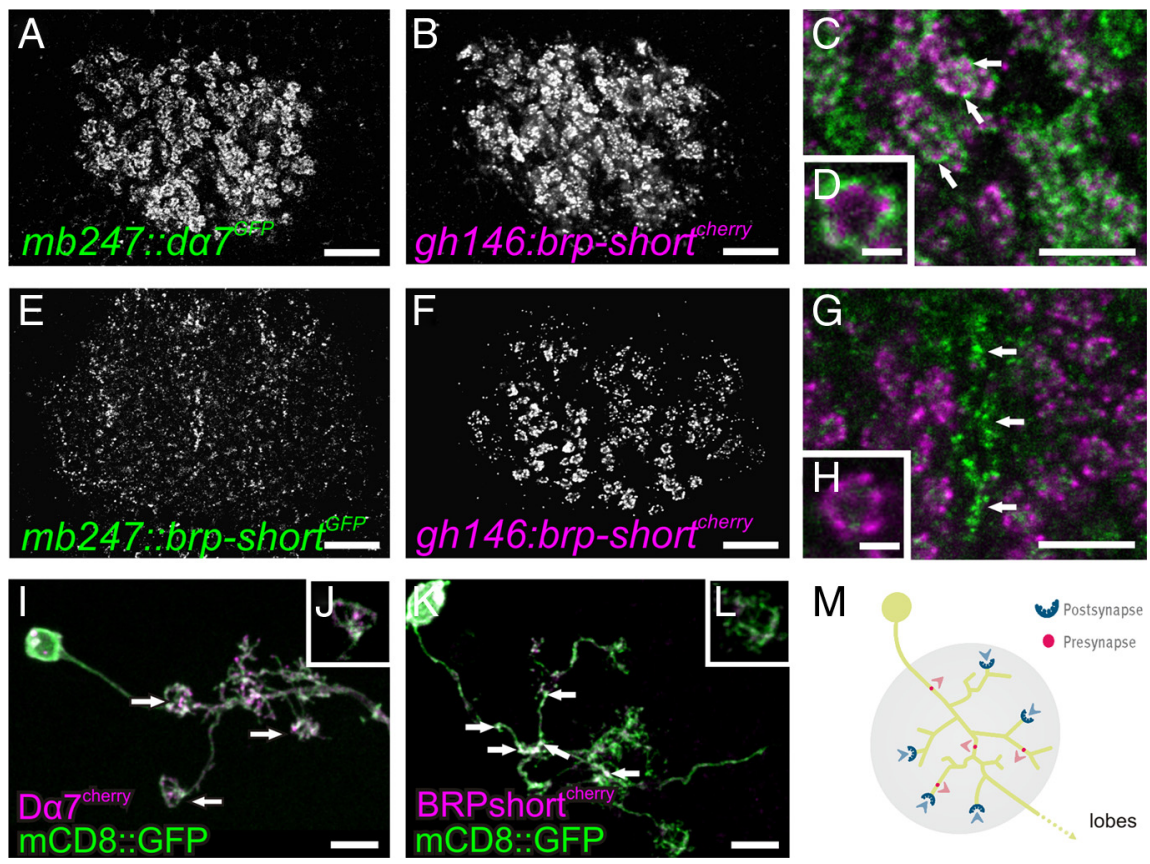

$\mathrm{M}$

Figure 6. Presynaptic and postsynaptic domains segregate within KC calyx dendrites. $\boldsymbol{A}-\boldsymbol{H}$, Analysis of synaptic elements in the calyx of adult flies. KCACs cluster distant from PN::KC synapses. A-D, Visualization of cholinergic PSDs of KCs $\left(m b 247-d \alpha 7^{G F P}\right)(\boldsymbol{A})$ together with PN-derived AZs ( $g$ h146-GAL4 driving UAS-brp-short ${ }^{\text {mcherry }}$ ) (B). A higher magnification of both merged labels shows that PN AZs locate at the inner edge of the microglomeruli, juxtaposed to KC PSDs ( $\boldsymbol{C}$, arrows; single microglomerulus in $\boldsymbol{D}$ ). $\boldsymbol{E}-\boldsymbol{H}$, In contrast, KC-expressed BRP-short (mb247::brp-short $\left.{ }^{G F P}\right)(\boldsymbol{E})$ clustered distant from PN AZ populations (marked by gh146::brp-short $\left.{ }^{\text {(herry }}\right)(\boldsymbol{F})$, as the merge of both labels at a higher magnification demonstrates ( $\boldsymbol{G}$, arrows; $\boldsymbol{H}$, single microglomerulus). $\boldsymbol{I}-\boldsymbol{L}$, Visualization of single KCs in the calyx using the MARCM technique. I, J, Single KC (maximum-intensity projection) expressing $\mathrm{D} \alpha 7^{\mathrm{mcherry}}$ together with $\mathrm{mCD8}$ ::GFP. A preferential localization of ACh receptors at the KC claw-like endings is visible ( $\boldsymbol{I}$, arrows; $\boldsymbol{J}$, single claw). $\boldsymbol{K}, \boldsymbol{L}$, Single KC (maximum-intensity projection) expressing BRP-short ${ }^{\text {mCherry }}$ together with mCD8::GFP. BRP signal is distributed along the $K C$ dendrites ( $\boldsymbol{K}$, arrows), mostly separated from the $K C$ claw-like endings ( $\boldsymbol{K}$, arrows; $\boldsymbol{L}$, single (law). $M$, Schematic drawing of synaptic input and output regions on a single $K C$ within the calyx. All images show single optical slices, except when stated differently. Slice thickness, $200 \mathrm{~nm}$. Scale bars: $\boldsymbol{A}, \boldsymbol{B}, \boldsymbol{E}, \boldsymbol{F}, 10 \mu \mathrm{m} ; \boldsymbol{C}, \boldsymbol{G}, \boldsymbol{I}, \boldsymbol{K}, 5 \mu \mathrm{m} ; \boldsymbol{D}, \boldsymbol{H}, 1 \mu \mathrm{m}$.

neurons [c739-GAL4 (O’Dell et al., 1995; Yang et al., 1995; Aso et al., 2009)], and $\alpha^{\prime} / \beta^{\prime}$ neurons [c305a-GAL4 (Krashes et al., 2007)] were used for coexpression of $U A S-d \alpha 7^{G F P}$ and $U A S$ brp-short ${ }^{\text {mCherry }}$ (Fig. 7D-L).

While expression in $\alpha^{\prime} / \beta^{\prime}$-type KCs produced a clear D $\alpha 7^{\text {GFP }}$ signal within the calyx, no BRP-short signals could be observed in the calyx (Fig. $7 D-F$ ) but in only $\alpha^{\prime} / \beta^{\prime}$ lobes (data not shown). The $\mathrm{D} \alpha 7^{\mathrm{GFP}}$ expression was strongest in four lateral units, whereas only a weak expression was observed in the center of the calyx (data not shown).

In $\gamma \mathrm{KCs}, \mathrm{D} \alpha 7$ but also BRP signals were observed in the calyx, with AZ and PSD signals being mainly segregated to distinct domains (Fig. 7G-I). The D $\alpha 7$ receptor construct labeled microglomeruli all over the calyx (Fig. $7 G$ ) but showed its strongest expression in the center as well as weaker levels in lateral regions of the calyx (data not shown). BRP-short preferentially localized around the $\mathrm{D} \alpha 7$-positive regions (Fig. 71 , arrows).

Also when expressed in $\alpha / \beta \mathrm{KCs}$, strong $\mathrm{D} \alpha 7$ and BRP-short signals were observed (Fig. $7 J-L$ ). As in the case of $\gamma \mathrm{KCs}$, AZs and PSDs derived from $\alpha / \beta$ KCs appeared segregated to mutually exclusive domains. The D $\alpha 7$ signal reproducibly labeled four units, residing laterally in the calyx (Fig. $7 \mathrm{M}-\mathrm{O}$ ). Closer analysis of image stacks identified four fascicles running along the cortex of the calyx (Fig. 7O, asterisks). These fascicles contained BRP when entering into the calyx, but contained $\mathrm{D} \alpha 7$ toward the center of the neuropil, with a border visible between both regions. It thus appears likely that individual $\alpha / \beta \mathrm{KC}$ dendrites consecu-

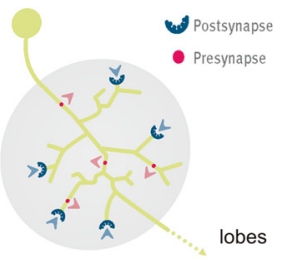

tively first form a proximal presynaptic specialization (BRP-positive AZs) followed by a more distal postsynaptic zone (D $\alpha 7$-positive PSDs).

Thus, only $\gamma$ and $\alpha / \beta$, but not $\alpha^{\prime} / \beta^{\prime}$ KCs seem to form KCACs. In an attempt to put these KC subtype-specific staining patterns in the context of the complete calyx, horizontal image stacks of adult calyces were examined. Here, BRPshort ${ }^{\text {mCherry }}$ was expressed under control of the PN driver gh146-GAL4 in addition to $m b 247:: d \alpha 7^{G F P}$ (to visualize the $\mathrm{PN}:$ :KC synapses) and a BRP ${ }^{\mathrm{Nc} 82}$ staining. We observed that the calyx appears subdivided into five subunits (Fig. $7 P$ ). Four of them were arranged in a pattern resembling a semicircle posterior to the inner antennocerebral tract (iACT), with the two lateral units terminating at the iACT. A fifth unit resided in between the semicycle. All five units harbored PN::KC synapses in their centers, which were surrounded by $\mathrm{BRP}^{\mathrm{Nc} 82}$-stained presynapses. We also analyzed the structure of the calyx with the previously mentioned KC-subset drivers (data not shown). The four laterally positioned subunits are mainly composed of $\alpha / \beta$ and $\alpha^{\prime} / \beta^{\prime}$ postsynapses, with $\alpha / \beta$ KCACs clustered at their outer edges. These four units, arranged in a closed semicircle, are responsible for the quadripartite appearance of the calyx, which has been described previously (Ito et al., 1997). The center of the fifth unit predominantly harbors the $\gamma$ neurons; their presynapses surround this unit and advance as far as in between the other four units. We summarized these findings in a tentative model (Fig. 7Q).

\section{Discussion}

In this study, we used several approaches to provide evidence that the $\mathrm{KC}$ dendrites within the calyx of larval and adult Drosophila are not exclusively postsynaptic. They also form presynaptic AZs, which we named KCACs. Our findings are supported by data from two previous studies (Rolls et al., 2007; Pauls et al., 2010), which reported the presence of a presynaptic vesicle protein, Synaptobrevin, in KCs within the calyx. Here, we show that KCACs are able to release SV s by a functional imaging approach. Furthermore, we examined which different KC subtypes form KCACs and provide a detailed description of the KCAC location within the calyx. The presence of these previously undescribed KCintrinsic presynaptic elements adds a new layer of complexity to the MB microcircuitry.

\section{KCACs and calycal microcircuitry}

Within KC dendrites, AZs and PSDs are clearly organized into discrete subdomains (Fig. 6I-M). Here, the question emerges whether a given $\mathrm{KC}$ dendrite is either exclusively presynaptic or postsynaptic, or whether both presynaptic and postsynaptic domains can be present within the same KC dendrite in a consecutive fashion. MARCM identified single KCs, which showed BRP puncta spatially segregated from claw-like regions that are 

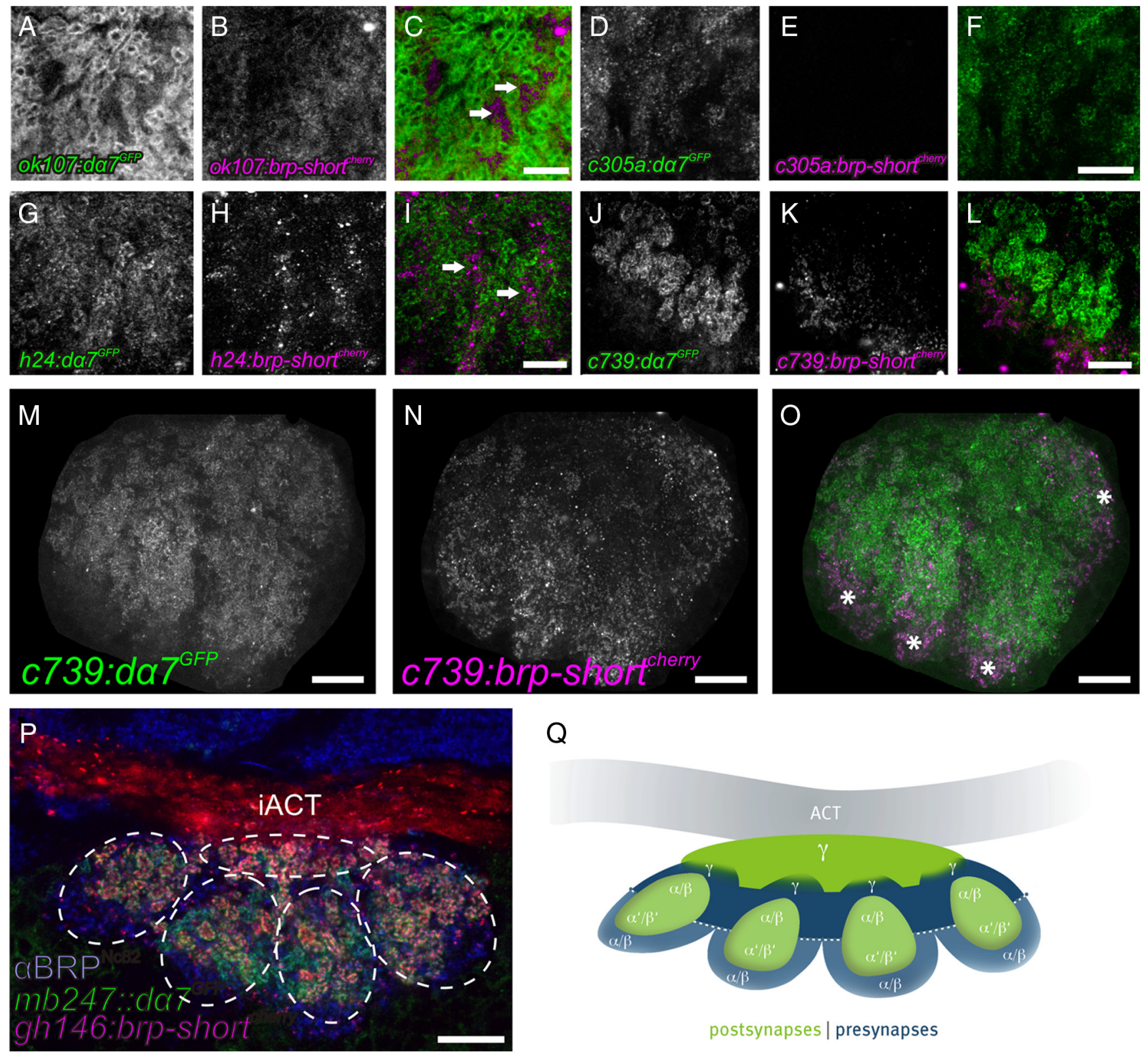

Q

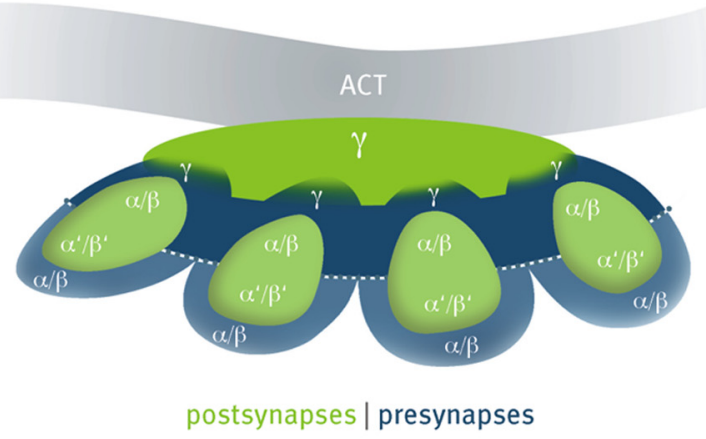

Figure 7. Coexpression of presynaptic (BRP-short) and postsynaptic ( $D \alpha 7)$ markers identifies distinct regions within KC dendrites of adult calyces. $A-L$ show cutouts of maximum-intensity projections of calyces. $\boldsymbol{A}-\boldsymbol{C}$, ok 107-GAL4 driving expression of both UAS- $d \alpha 7^{G F P}(\boldsymbol{A})$ and UAS-brp-short ${ }^{\text {mCherry }}(\boldsymbol{B})$ within KC dendrites. The merged image shows that presynaptic and postsynaptic regions appeared largely segregated (C, arrows). D-F, $\alpha^{\prime} \beta^{\prime}$ KCs (C305a-GAL4) expressing both UAS-d $\alpha 7^{G F P}(\boldsymbol{D})$ and UAS-brp-short ${ }^{\text {mCherry }}$ (E). While a strong D $\alpha 7$ signal was present (D), no BRP-short signal could be detected within the calyx $(\boldsymbol{E}, \boldsymbol{F}) . \mathbf{G}-\boldsymbol{I}, \gamma \mathrm{KC}\left(\right.$ h24-GAL4) expressing both UAS- $d \alpha 7^{G F P}(\boldsymbol{G})$ and UAS-brp-short ${ }^{m \text { Cherry }}(\boldsymbol{H})$. Both signals were present and clearly separated from each other $(\boldsymbol{I})$. D $\alpha 7$ was distributed in microglomerular structures, while the BRP-short signal localized in between (I, arrows). J-0, $\alpha \beta \mathrm{KCS}\left(\mathrm{C} 739\right.$-GAL4) expressing both UAS-d $\alpha 7^{6 F P}(\boldsymbol{J}, \boldsymbol{M})$ and UAS-brp-short ${ }^{\text {mCherry }}(\boldsymbol{K}, \boldsymbol{N}) \cdot \boldsymbol{M}-\mathbf{O}$ show the whole calyx (maximum-intensity projection). The dendrites showed a similar segregation of presynaptic and postsynaptic regions $(\boldsymbol{L}, \mathbf{0})$. Both signals were arranged into four distinct patches clearly separated from each other $(\boldsymbol{O}$, asterisks). Here, BRP-short was located at the more peripheral part of the calyx $(\boldsymbol{K}, \boldsymbol{N})$, whereas $D \alpha 7$ showed a stronger signal toward the center of the neuropil $(\boldsymbol{J}, \boldsymbol{M})$.P. , Horizontal section through the calyx of an adult fly. Visualization of calycal microglomeruli by UAS-brp-short ${ }^{\text {mCherry }}$ expressed in PNs ( $g$ h 146-GAL4) and $\mathrm{D} \alpha 7$ expressed in $\mathrm{KCs}\left(\mathrm{mb} 247:: \mathrm{d} \alpha 7^{G \mathrm{FP}}\right)$. A $\alpha \mathrm{BRP} \mathrm{P}^{\mathrm{N} 82}$ label of all presynapses is shown in blue. The calyx is divided into five subunits, each harboring microglomeruli in the center, that are surrounded by other synapses. Four subunits protrude to the posterior part and one further subunit is located between them and the iACT. $\boldsymbol{Q}$, Schematic drawing of the distribution of presynaptic and postsynaptic regions of the different $K C$ subtypes within the MB calyx. Postsynapses are shown in green, and presynapses are shown in blue. All images show single optical slices, except when stated differently. Slice thickness, $200 \mathrm{~nm}$. iACT, Inner antennocerebral tract. Scale bars: $\boldsymbol{C}, \boldsymbol{F}, \boldsymbol{I}, \boldsymbol{L}, 5 \mu \mathrm{m} ; \boldsymbol{M}-\boldsymbol{P}, 10 \mu \mathrm{m}$. BRP-short can form agglomerations within the somata of the cells it is expressed in. As the KC somata are located very close to the calyx neuropil, we manually removed the somata from images $\boldsymbol{M}-\mathbf{O}$ for clarity.

thought to harbor the postsynaptic specializations of cholinergic $\mathrm{PN}:: \mathrm{KC}$ synapses. In parallel experiments, we showed that these claws in fact clustered the acetylcholine receptor $\mathrm{D} \alpha 7$, as was expected from our previous work (Kremer et al., 2010). Thus, it appears likely that presynaptic and postsynaptic domains can be present within the same KC dendrite.
Based on the BRP-RNAi analysis, we estimate that $\sim 20-30 \%$ of all presynapses in the calyx are KCACs, in both adults and larvae. These synapses are apparently part of the general calyx microcircuitry. They might synapse onto PNs, KCs themselves, the anterior paired lateral (APL) neuron, modulatory neurons, or so-far-undescribed cells. From our analysis, it appears unlikely 
that PN boutons are direct postsynaptic partners of the KCACs, as the KCACs appear to be clearly physically segregated from the PN boutons. KCACs might, however, project onto PN axons.

It appears well possible that KCACs project onto the GABAergic APL neuron (Liu and Davis, 2009), which arborizes in the whole calyx. Within the insect antennal lobe, reciprocal dendrodendritic connections between the PNs and the partially GABAergic local interneurons (LNs) have been described (Sun et al., 1997; Didier et al., 2001; Ng et al., 2002). The PN neurites and the LNs are both transmissive and receptive in the antennal lobe, suggesting a computation between them. KCACs might be involved into similar computations in the calyx. This would be in accordance with EM studies in crickets that suggest presynapses in KCs that connect to GABAergic fibers in the MB calyx (Schürmann et al., 2008).

KCACs might also mediate KC::KC communication. In fact, dendritic segments of KCs that harbor presynapses appear to run in a parallel fashion (data not shown). This arrangement could promote the communication between dendritic segments of KCs via dendrodendritic synapses. Such KC::KC synapses could therefore modulate signals originating from the distal segments of the arborizations, which carry odor-evoked signals. By these means, an effective computation between KCs could be accomplished before they transmit their input signals downstream.

Unfortunately, at the moment no general PSD markers are available in Drosophila. Moreover, the neurotransmitter used by KCs remains unknown. With a general postsynaptic marker or knowledge about the KC transmitter, we could have generated tools to identify the postsynaptic partners of KCACs. Yet currently, despite efforts, we can only speculate about the postsynaptic partners of KCs in the calyx.

\section{Potential roles of KCACs in learning and memory processes}

Memory traces are typically thought to be manifested as plastic changes in neuronal anatomy and physiology that occur in specific brain regions. Several lines of evidence indicate that MBs are causally involved in associative learning of olfactory stimuli (for review, see Heisenberg, 1998, 2003; Roman and Davis, 2001; Waddell and Quinn, 2001b; Dubnau et al., 2003; Gerber et al., 2004; Davis, 2005; Keene and Waddell, 2007). Flies with chemically ablated KCs or mutants lacking the MBs are deficient in olfactory learning (Heisenberg et al., 1985; de Belle and Heisenberg, 1994). Learning was investigated in flies mutant for the adenylyl cyclase rutabaga ( $r u t$ ) (Livingstone et al., 1984), which is suggested to act as a coincidence detector between conditioned stimulus (odor) and unconditioned stimulus (e.g., electric shock) (Tomchik and Davis, 2009). Reexpression of a rut cDNA in a rut $^{-}$background within a subpopulation of KCs sufficed to restore odor learning (Zars et al., 2000; McGuire et al., 2003). For appetitive learning, reexpression of rut in either PNs or KCs is sufficient to restore the mutant defect, whereas aversive learning is rescued only by rut reexpression in KCs (Thum et al., 2007). Reversible disruption of transmitter release in Drosophila KCs, using a temperature-sensitive dynamin transgene, UAS-shibire ${ }^{t s 1}$ (Kitamoto, 2001), was shown to block memory retrieval in $\alpha / \beta$ neurons (McGuire et al., 2001; Isabel et al., 2004; Keene and Waddell, 2007; Krashes et al., 2007) and acquisition and stabilization of memory in $\alpha^{\prime} / \beta^{\prime}$ neurons (Keene and Waddell, 2007; Krashes et al., 2007). Together, these data imply that MBs play a major role in learning and memory. To form, stabilize, and retrieve memory (for review, see Keene and Waddell, 2007), KCs use their presynapses (for review, see Heisenberg, 2003; Davis,
2005; Keene and Waddell, 2007). The KC presynapses are so far believed to reside in the lobes.

The biogenic amines octopamine and dopamine are thought to mediate the unconditioned stimulus signal for learning olfactory associations, with octopamine representing appetitive stimuli and dopamine representing aversive stimuli (Schwaerzel et al., 2003; Schroll et al., 2006). Hammer and Menzel (1998) could show that, in honeybees, sugar can be replaced by octopamine application to the calyx to trigger the conditioned proboscisextension reflex. In the fruit fly, the amines octopamine and dopamine are released onto MB lobes and calyx (Sinakevitch and Strausfeld, 2006; Busch et al., 2009; Mao and Davis, 2009). This holds also true for the larva [octopamine (A. Thum, personal communication); dopamine (Selcho et al., 2009)]. Therefore, the KCACs might be involved in appetitive learning as well as in aversive learning in fly and larva.

Notably, we found that the KC subpopulations $\gamma$ and $\alpha / \beta$, but not $\alpha^{\prime} / \beta^{\prime}$, form KCACs (Fig. 7). This dichotomy correlates with functional differences in learning and memory that have been assigned to these $\mathrm{KC}$ classes in previous studies. For example, $\alpha^{\prime} / \beta^{\prime}$ KCs were reported to be required during and after training to acquire and stabilize olfactory memory (Keene and Waddell, 2007; Krashes et al., 2007), whereas output from $\alpha / \beta$ neurons was postulated to be required to retrieve memory (McGuire et al., 2001; Keene and Waddell, 2007; Krashes et al., 2007). It has been proposed that, during acquisition, olfactory information received from PNs is first processed in parallel by the $\alpha / \beta$ and $\alpha^{\prime} / \beta^{\prime}$ KCs (Keene and Waddell, 2007; Krashes et al., 2007). Notably, activity in $\alpha^{\prime} / \beta^{\prime} \mathrm{KCs}$ (which do not form KCACs) is supposed to trigger a recurrent loop between $\alpha^{\prime} / \beta^{\prime}$ KCs and dorsal paired medial neurons, which project to the MB lobes (Yu et al., 2005; Keene et al., 2006; Keene and Waddell, 2007; Krashes et al., 2007). This loop, in turn, might be necessary for memory consolidation in $\alpha / \beta$ neurons. Subsequently, memories could be stored in $\alpha / \beta$ neurons, whose activity is required during recall (Keene and Waddell, 2007; Krashes et al., 2007). As $\alpha^{\prime} / \beta^{\prime}$ neurons are devoid of KCACs, KCACs cannot be involved in the circuit described above. Instead, it is likely that additional, similar recurrent loops exist, which are mediated via KCACs. However, it remains unresolved how exactly $\mathrm{KC}: \mathrm{KC}$ communication is organized anatomically and functionally. Our study now proposes a newly discovered synapse population as candidate sites for $\mathrm{KC}: \mathrm{KC}$ communication.

In the mammalian olfactory system, major feedback pathways exist, which project onto neurons one level lower in hierarchy (Haberly, 1998). A recent publication (Hu et al., 2010) showed that likewise in Drosophila activation of KCs induced a depolarization in cell bodies of PNs and LNs within the antennal lobes. The authors thus suggested that MB lobes provide feedback to the ALs. Moreover, an additional memory trace appears to exist in the antennal lobe (Hammer and Menzel, 1995, 1998; Faber et al., 1999; Yu et al., 2004)/in the PNs (Thum et al., 2007). It may therefore well be the case that KCs project onto PNs or onto feedback neurons via their KCACs.

An urgent question of the field concerns the identification of the postsynaptic partner cells of KC presynapses, which harbor memory traces during olfactory conditioning. It is generally assumed that MB-extrinsic downstream neurons involved in behavioral execution of learned behavior serve as postsynaptic partners here. Our findings raise the possibility that microcircuits inside the MB could be places for further modulation and computation of olfactory processing and/or memory formation and modulation. As a consequence, not only the communication to 
downstream neurons but also the representation of sensory information within the MB circuitry might be changed by experience. Future analysis using optophysiological tools at the KCACs, together with further anatomical work, should provide answers to these questions.

\section{References}

Akalal DB, Wilson CF, Zong L, Tanaka NK, Ito K, Davis RL (2006) Roles for Drosophila mushroom body neurons in olfactory learning and memory. Learn Mem 13:659-668.

Aso Y, Grübel K, Busch S, Friedrich AB, Siwanowicz I, Tanimoto H (2009) The mushroom body of adult Drosophila characterized by GAL4 drivers. J Neurogenet 23:156-172.

Aso Y, Siwanowicz I, Bräcker L, Ito K, Kitamoto T, Tanimoto H (2010) Specific dopaminergic neurons for the formation of labile aversive memory. Curr Biol 20:1445-1451.

Busch S, Selcho M, Ito K, Tanimoto H (2009) A map of octopaminergic neurons in the Drosophila brain. J Comp Neurol 513:643-667.

Claridge-Chang A, Roorda RD, Vrontou E, Sjulson L, Li H, Hirsh J, Miesenböck G (2009) Writing memories with light-addressable reinforcement circuitry. Cell 139:405-415.

Connolly JB, Roberts IJ, Armstrong JD, Kaiser K, Forte M, Tully T, O'Kane CJ (1996) Associative learning disrupted by impaired Gs signaling in Drosophila mushroom bodies. Science 274:2104-2107.

Crittenden JR, Skoulakis EM, Han KA, Kalderon D, Davis RL (1998) Tripartite mushroom body architecture revealed by antigenic markers. Learn Mem 5:38-51.

Davis RL (2004) Olfactory learning. Neuron 44:31-48.

Davis RL (2005) Olfactory memory formation in Drosophila: from molecular to systems neuroscience. Annu Rev Neurosci 28:275-302.

de Belle JS, Heisenberg M (1994) Associative odor learning in Drosophila abolished by chemical ablation of mushroom bodies. Science 263:692-695.

Didier A, Carleton A, Bjaalie JG, Vincent JD, Ottersen OP, Storm-Mathisen J, Lledo PM (2001) A dendrodendritic reciprocal synapse provides a recurrent excitatory connection in the olfactory bulb. Proc Natl Acad Sci U S A 98:6441-6446.

Dubnau J, Chiang AS, Tully T (2003) Neural substrates of memory: from synapse to system. J Neurobiol 54:238-253.

Faber T, Joerges J, Menzel R (1999) Associative learning modifies neural representations of odors in the insect brain. Nat Neurosci 2:74-78.

Fayyazuddin A, Zaheer MA, Hiesinger PR, Bellen HJ (2006) The nicotinic acetylcholine receptor Dalpha7 is required for an escape behavior in Drosophila. PLoS Biol 4:e63.

Gerber B, Tanimoto H, Heisenberg M (2004) An engram found? Evaluating the evidence from fruit flies. Curr Opin Neurobiol 14:737-744.

Grauso M, Reenan RA, Culetto E, Sattelle DB (2002) Novel putative nicotinic acetylcholine receptor subunit genes, Dalpha5, Dalpha6 and Dalpha7, in Drosophila melanogaster identify a new and highly conserved target of adenosine deaminase acting on RNA-mediated A-to-I premRNA editing. Genetics 160:1519-1533.

Haberly LB (1998) Olfactory cortex. In: The synaptic organization of the brain (Shepherd GM, ed), pp 317-345. New York: Oxford UP.

Hammer M, Menzel R (1995) Learning and memory in the honeybee. J Neurosci 15:1617-1630.

Hammer M, Menzel R (1998) Multiple sites of associative odor learning as revealed by local brain microinjections of octopamine in honeybees. Learn Mem 5:146-156.

Heisenberg M (1998) What do the mushroom bodies do for the insect brain? An introduction. Learn Mem 5:1-10.

Heisenberg M (2003) Mushroom body memoir: from maps to models. Nat Rev Neurosci 4:266-275.

Heisenberg M, Borst A, Wagner S, Byers D (1985) Drosophila mushroom body mutants are deficient in olfactory learning. J Neurogenet 2:1-30.

Hu A, Zhang W, Wang Z (2010) Functional feedback from mushroom bodies to antennal lobes in the Drosophila olfactory pathway. Proc Natl Acad Sci U S A 107:10262-10267.

Isabel G, Pascual A, Preat T (2004) Exclusive consolidated memory phases in Drosophila. Science 304:1024-1027.

Ito K, Awano W, Suzuki K, Hiromi Y, Yamamoto D (1997) The Drosophila mushroom body is a quadruple structure of clonal units each of which contains a virtually identical set of neurones and glial cells. Development 124:761-771.

Joiner WJ, Crocker A, White BH, Sehgal A (2006) Sleep in Drosophila is regulated by adult mushroom bodies. Nature 441:757-760.

Keene AC, Waddell S (2007) Drosophila olfactory memory: single genes to complex neural circuits. Nat Rev Neurosci 8:341-354.

Keene AC, Krashes MJ, Leung B, Bernard JA, Waddell S (2006) Drosophila dorsal paired medial neurons provide a general mechanism for memory consolidation. Curr Biol 16:1524-1530.

Kitamoto T (2001) Conditional modification of behavior in Drosophila by targeted expression of a temperature-sensitive shibire allele in defined neurons. J Neurobiol 47:81-92.

Kittel RJ, Wichmann C, Rasse TM, Fouquet W, Schmidt M, Schmid A, Wagh DA, Pawlu C, Kellner RR, Willig KI, Hell SW, Buchner E, Heckmann M, Sigrist SJ (2006) Bruchpilot promotes active zone assembly, $\mathrm{Ca}^{2+}$ channel clustering, and vesicle release. Science 312:1051-1054.

Krashes MJ, Keene AC, Leung B, Armstrong JD, Waddell S (2007) Sequential use of mushroom body neuron subsets during Drosophila odor memory processing. Neuron 53:103-115.

Krashes MJ, DasGupta S, Vreede A, White B, Armstrong JD, Waddell S (2009) A neural circuit mechanism integrating motivational state with memory expression in Drosophila. Cell 139:416-427.

Kremer MC, Christiansen F, Leiss F, Paehler M, Knapek S, Andlauer TF, Förstner F, Kloppenburg P, Sigrist SJ, Tavosanis G (2010) Structural long-term changes at mushroom body input synapses. Curr Biol 20:1938-1944.

Lee T, Luo L (2001) Mosaic analysis with a repressible cell marker (MARCM) for Drosophila neural development. Trends Neurosci 24:251-254

Lee T, Lee A, Luo L (1999) Development of the Drosophila mushroom bodies: sequential generation of three distinct types of neurons from a neuroblast. Development 126:4065-4076.

Leiss F, Koper E, Hein I, Fouquet W, Lindner J, Sigrist S, Tavosanis G (2009) Characterization of dendritic spines in the Drosophila central nervous system. Dev Neurobiol 69:221-234.

Liu L, Wolf R, Ernst R, Heisenberg M (1999) Context generalization in Drosophila visual learning requires the mushroom bodies. Nature 400:753-756.

Liu X, Davis RL (2009) The GABAergic anterior paired lateral neuron suppresses and is suppressed by olfactory learning. Nat Neurosci 12:53-59.

Livingstone MS, Sziber PP, Quinn WG (1984) Loss of calcium/calmodulin responsiveness in adenylate cyclase of rutabaga, a Drosophila learning mutant. Cell 37:205-215.

Luo L (2007) Fly MARCM and mouse MADM: genetic methods of labeling and manipulating single neurons. Brain Res Rev 55:220-227.

Mao Z, Davis RL (2009) Eight different types of dopaminergic neurons innervate the Drosophila mushroom body neuropil: anatomical and physiological heterogeneity. Front Neural Circuits 3:5.

McBride SM, Giuliani G, Choi C, Krause P, Correale D, Watson K, Baker G, Siwicki KK (1999) Mushroom body ablation impairs short-term memory and long-term memory of courtship conditioning in Drosophila melanogaster. Neuron 24:967-977.

McGuire SE, Le PT, Davis RL (2001) The role of Drosophila mushroom body signaling in olfactory memory. Science 293:1330-1333.

McGuire SE, Le PT, Osborn AJ, Matsumoto K, Davis RL (2003) Spatiotemporal rescue of memory dysfunction in Drosophila. Science 302:1765-1768.

Miesenböck G, De Angelis DA, Rothman JE (1998) Visualizing secretion and synaptic transmission with $\mathrm{pH}$-sensitive green fluorescent proteins. Nature 394:192-195.

Miller PM, Saltz JB, Cochrane VA, Marcinkowski CM, Mobin R, Turner TL (2011) Natural variation in decision-making behavior in Drosophila melanogaster. PLoS One 6:e16436.

Mombaerts P (2001) How smell develops. Nat Neurosci 4 [Suppl]:1192-1198.

Ng M, Roorda RD, Lima SQ, Zemelman BV, Morcillo P, Miesenböck G (2002) Transmission of olfactory information between three populations of neurons in the antennal lobe of the fly. Neuron 36:463-474.

O'Dell KM, Armstrong JD, Yang MY, Kaiser K (1995) Functional dissection of the Drosophila mushroom bodies by selective feminization of genetically defined subcompartments. Neuron 15:55-61.

Owald D, Fouquet W, Schmidt M, Wichmann C, Mertel S, Depner H, Chris- 
tiansen F, Zube C, Quentin C, Körner J, Urlaub H, Mechtler K, Sigrist SJ (2010) A Syd-1 homologue regulates pre- and postsynaptic maturation in Drosophila. J Cell Biol 188:565-579.

Pauls D, Selcho M, Gendre N, Stocker RF, Thum AS (2010) Drosophila larvae establish appetitive olfactory memories via mushroom body neurons of embryonic origin. J Neurosci 30:10655-10666.

Pitman JL, McGill JJ, Keegan KP, Allada R (2006) A dynamic role for the mushroom bodies in promoting sleep in Drosophila. Nature 441:753-756.

Prokop A, Meinertzhagen IA (2006) Development and structure of synaptic contacts in Drosophila. Semin Cell Dev Biol 17:20-30.

Raghu SV, Joesch M, Sigrist SJ, Borst A, Reiff DF (2009) Synaptic organization of lobula plate tangential cells in Drosophila: Dalpha7 cholinergic receptors. J Neurogenet 23:200-209.

Ramaekers A, Magnenat E, Marin EC, Gendre N, Jefferis GS, Luo L, Stocker RF (2005) Glomerular maps without cellular redundancy at successive levels of the Drosophila larval olfactory circuit. Curr Biol 15:982-992.

Riemensperger T, Völler T, Stock P, Buchner E, Fiala A (2005) Punishment prediction by dopaminergic neurons in Drosophila. Curr Biol 15:1953-1960.

Rolls MM, Satoh D, Clyne PJ, Henner AL, Uemura T, Doe CQ (2007) Polarity and intracellular compartmentalization of Drosophila neurons. Neural Dev 2:7.

Roman G, Davis RL (2001) Molecular biology and anatomy of Drosophila olfactory associative learning. Bioessays 23:571-581.

Schmid A, Hallermann S, Kittel RJ, Khorramshahi O, Frölich AM, Quentin C, Rasse TM, Mertel S, Heckmann M, Sigrist SJ (2008) Activity-dependent site-specific changes of glutamate receptor composition in vivo. Nat Neurosci 11:659-666.

Schroll C, Riemensperger T, Bucher D, Ehmer J, Völler T, Erbguth K, Gerber B, Hendel T, Nagel G, Buchner E, Fiala A (2006) Light-induced activation of distinct modulatory neurons triggers appetitive or aversive learning in Drosophila larvae. Curr Biol 16:1741-1747.

Schulz RA, Chromey C, Lu MF, Zhao B, Olson EN (1996) Expression of the D-MEF2 transcription in the Drosophila brain suggests a role in neuronal cell differentiation. Oncogene 12:1827-1831.

Schürmann FW (1974) On the functional anatomy of the corpora pedunculata in insects (in German). Exp Brain Res 19:406-432.

Schürmann FW, Frambach I, Elekes K (2008) GABAergic synaptic connections in mushroom bodies of insect brains. Acta Biol Hung 59 [Suppl]:173-181.

Schwaerzel M, Monastirioti M, Scholz H, Friggi-Grelin F, Birman S, Heisenberg M (2003) Dopamine and octopamine differentiate between aversive and appetitive olfactory memories in Drosophila. J Neurosci 23:10495-10502.

Selcho M, Pauls D, Han KA, Stocker RF, Thum AS (2009) The role of dopamine in Drosophila larval classical olfactory conditioning. PLoS One 4:e5897.

Sigrist SJ, Reiff DF, Thiel PR, Steinert JR, Schuster CM (2003) Experiencedependent strengthening of Drosophila neuromuscular junctions. J Neurosci 23:6546-6556.

Sinakevitch I, Strausfeld NJ (2006) Comparison of octopamine-like immunoreactivity in the brains of the fruit fly and blow fly. J Comp Neurol 494:460-475.
Stocker RF, Singh RN, Schorderet M, Siddiqi O (1983) Projection patterns of different types of antennal sensilla in the antennal glomeruli of Drosophila melanogaster. Cell Tissue Res 232:237-248.

Stocker RF, Heimbeck G, Gendre N, de Belle IS (1997) Neuroblast ablation in Drosophila P[GAL4] lines reveals origins of olfactory interneurons. J Neurobiol 32:443-456.

Strausfeld NJ (1976) Atlas of the insect brain. Berlin: Springer.

Sun XJ, Tolbert LP, Hildebrand JG (1997) Synaptic organization of the uniglomerular projection neurons of the antennal lobe of the moth Manduca sexta: a laser scanning confocal and electron microscopic study. J Comp Neurol 379:2-20.

Thum AS, Jenett A, Ito K, Heisenberg M, Tanimoto H (2007) Multiple memory traces for olfactory reward learning in Drosophila. J Neurosci 27:11132-11138.

Tomchik SM, Davis RL (2009) Dynamics of learning-related cAMP signaling and stimulus integration in the Drosophila olfactory pathway. Neuron 64:510-521.

Waddell S, Quinn WG (2001a) Flies, genes, and learning. Annu Rev Neurosci 24:1283-1309.

Waddell S, Quinn WG (2001b) What can we teach Drosophila? What can they teach us? Trends Genet 17:719-726.

Waddell S, Armstrong JD, Kitamoto T, Kaiser K, Quinn WG (2000) The amnesiac gene product is expressed in two neurons in the Drosophila brain that are critical for memory. Cell 103:805-813.

Wagh DA, Rasse TM, Asan E, Hofbauer A, Schwenkert I, Dürrbeck H, Buchner S, Dabauvalle MC, Schmidt M, Qin G, Wichmann C, Kittel R, Sigrist SJ, Buchner E (2006) Bruchpilot, a protein with homology to ELKS/ CAST, is required for structural integrity and function of synaptic active zones in Drosophila. Neuron 49:833-844.

Wichmann C, Sigrist SJ (2010) The active zone T-bar-a plasticity module? J Neurogenet 24:133-145.

Yang CH, Belawat P, Hafen E, Jan LY, Jan YN (2008) Drosophila egg-laying site selection as a system to study simple decision-making processes. Science 319:1679-1683.

Yang MY, Armstrong JD, Vilinsky I, Strausfeld NJ, Kaiser K (1995) Subdivision of the Drosophila mushroom bodies by enhancer-trap expression patterns. Neuron 15:45-54.

Yasuyama K, Meinertzhagen IA, Schürmann FW (2002) Synaptic organization of the mushroom body calyx in Drosophila melanogaster. J Comp Neurol 445:211-226.

Yu D, Ponomarev A, Davis RL (2004) Altered representation of the spatial code for odors after olfactory classical conditioning; memory trace formation by synaptic recruitment. Neuron 42:437-449.

Yu D, Keene AC, Srivatsan A, Waddell S, Davis RL (2005) Drosophila DPM neurons form a delayed and branch-specific memory trace after olfactory classical conditioning. Cell 123:945-957.

Zars T, Fischer M, Schulz R, Heisenberg M (2000) Localization of a shortterm memory in Drosophila. Science 288:672-675.

Zhang YQ, Rodesch CK, Broadie K (2002) Living synaptic vesicle marker: synaptotagmin-GFP. Genesis 34:142-145. 